\title{
Communication-Aware Task Scheduling Strategy in Hybrid MPI+OpenMP Applications
}

\author{
Romain Pereira ${ }^{1,3}$, Adrien Roussel ${ }^{1,2}$, Patrick Carribault ${ }^{1,2}$, and Thierry \\ Gautier $^{3}$ \\ 1 CEA, DAM, DIF, F-91297 Arpajon, France \\ \{romain.pereira, adrien.roussel, patrick.carribault\}@cea.fr \\ 2 Université Paris-Saclay, CEA, Laboratoire en Informatique Haute Performance \\ pour le Calcul et la simulation, 91680 Bruyères-le-Châtel, France \\ 3 Project Team AVALON INRIA, LIP, ENS-Lyon, Lyon, France \\ thierry.gautier@inrialpes.fr
}

\begin{abstract}
While task-based programming, such as OpenMP, is a promising solution to exploit large HPC compute nodes, it has to be mixed with data communications like MPI. However, performance or even more thread progression may depend on the underlying runtime implementations. In this paper, we focus on enhancing the application performance when an OpenMP task blocks inside MPI communications. This technique requires no additional effort on the application developers. It relies on an online task re-ordering strategy that aims at running first tasks that are sending data to other processes. We evaluate our approach on a Cholesky factorization and show that we gain around $19 \%$ of execution time on an Intel Skylake compute nodes machine - each node having two 24-core processors.
\end{abstract}

Keywords: MPI + OpenMP · Task · Scheduling · Asynchronism

\section{Introduction}

High Performance Computing (HPC) applications target distributed machines, which inevitably involve inter-node data exchanges that can be handled by MPI (Message Passing Interface). But, at compute-node level, the number of cores is increasing, and task programming models seem to be well-suited for efficient use of all computing resources and to satisfy the needs of asynchronism. Since 2008, OpenMP $[1,14]$ defines a standard for task programming. This leads to codes that finely nest MPI communications within such OpenMP tasks. Furthermore, OpenMP 4.0 introduced data dependencies between tasks. Thus, within parallel regions exploiting tasks, applications can be seen as a single global data-flow graph which is distributed across MPI processes, where each process has its own OpenMP task scheduler with no view of the global graph. This may result in poor performance [12] and even deadlocks [19].

Deadlocks can be due to the loss of cores when threads execute blocking MPI calls within OpenMP tasks [11]. Several solutions address this issue $[16,18,20]$ and enable working MPI + OpenMP(tasks) codes, but performance issues remain. 


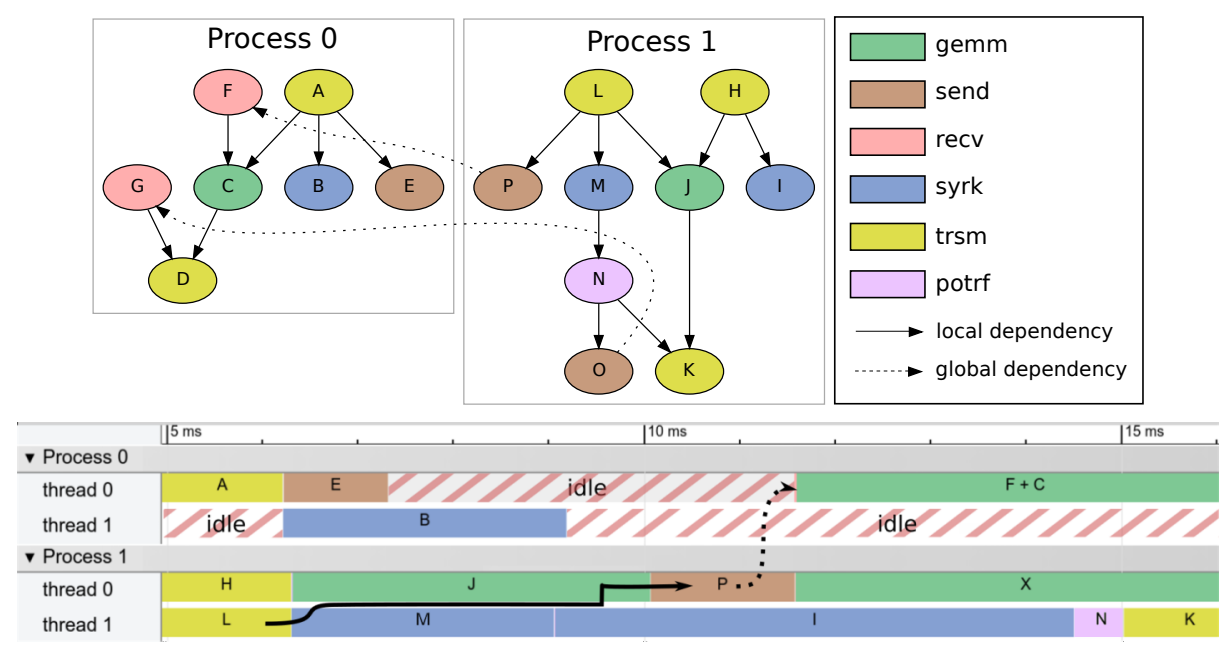

Fig. 1. Top: sub-graph of a distributed blocked Cholesky factorization mapped onto 2 MPI Ranks (matrix size: 2048x2048 with tile of size 512). Bottom: Gantt chart with 2 threads per MPI rank.

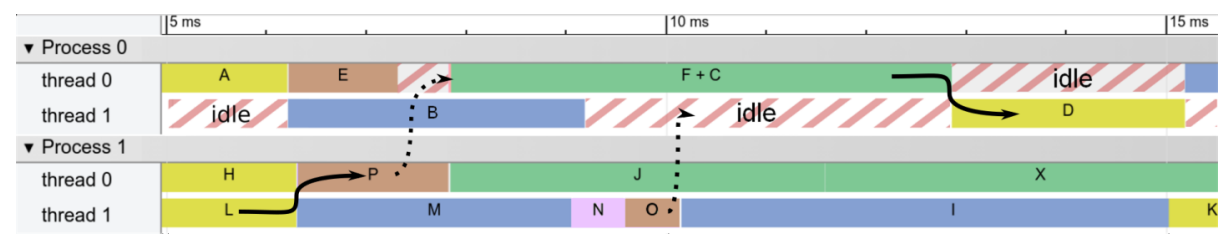

Fig. 2. Alternative scheduling for the graph in Figure 1.

Task scheduling in this hybrid context can significantly improve the overall performance. As an example, Figure 1 presents a subgraph of the task dependency graph (TDG) for a Cholesky factorization [19], and its scheduling trace on 2 processors of 2 threads each. Tasks are scheduled following a standard First In, First Out (FIFO) policy: once precedence constraints are resolved, the first tasks created are scheduled first. This policy leads to $61 \%$ idle time on Process 0, partially because it is waiting for data from Process 1. A similar result would be obtained with regular OpenMP runtimes (GNU-OpenMP [6], LLVM-OpenMP [8]) which uses a mix of FIFO and Last In, First Out (LIFO) policies. It is possible to reduce this idle time to $36 \%$ by adapting the scheduling policy to prioritize inter-process edges (see Figure 2).

This paper proposes a communication-aware task re-ordering strategy for OpenMP that aims at reducing idle periods in hybrid MPI+OpenMP (tasks) applications by favoring tasks on a path to communications. This strategy relies on hybrid scheduling techniques and proposes an automatic TDG prioritization based on communication information. Our solution heavily 
leverages runtime interoperations but requires no OpenMP / MPI extensions and no further efforts on the user side on tasks prioritization. Section 2 presents related work. Then, Section 3 highlights our task scheduling strategy and Section 4 exposes its implementation and evaluation. Finally, Section 5 concludes and discusses future work.

\section{Related Work}

The Dominant Sequence Clustering (DSC) [22] was proposed as a heuristic for scheduling tasks on an unlimited number of distributed cores. This algorithm distributes a fully-discovered TDG onto cores - the clustering phase - and prioritizes tasks using global bottom and top levels. Our paper focuses on hybrid MPI + OpenMP(tasks) which are the widely used standards in the HPC community. With this programming model, the clustering is done by the user which distributes OpenMP tasks onto MPI processes. Each OpenMP tasks scheduler only has a view on its local subgraph (i.e. its cluster). The tasks global bottom and top levels (blevel, tlevel) are not known, which leads us to prioritize the TDG using purely local information.

MPI + OpenMP(tasks) model may lead to a loss of thread, when a thread executes blocking MPI code within an OpenMP task [11,12]. Many works addressed this issue $[4,9,11,16,18,19]$. Some approaches $[4,11]$ consist of marking communication tasks from user codes and dedicating threads to communication or computation. This guarantees that both communications and computations progress and fix deadlock issues due to the loss of threads. However, it requires user-code adaptations and creates load balancing issues between communication and computation threads. In 2015, MPI+ULT (User Level Thread, Argobots) [9] proposed to run MPI code within a user-level thread, and make it yield whenever an MPI communication blocks. In OpenMP, yielding can be achieved using the taskyield directive. Schuchart et al [19] explored various implementations of it, and having implementations that effectively suspend, enables the expression of fine MPI data movement within OpenMP tasks. This resulted in a more efficient implementation of the blocked Cholesky factorization with fewer synchronizations and led to new approaches on MPI+OpenMP(tasks) interoperability, such as TAMPI [18] and MPI_Detach [16]. TAMPI was proposed as a user library to enable blocking-tasks pause and resume mechanism. It transforms calls to MPI blocking operations to non-blocking ones through the PMPI interface and interoperates with the underlying tasking runtime - typically using the taskyield in OpenMP, or nanos6_block_current_task in Nanos6. The authors of [16] proposed another interoperability approach using the detach clause, which implies MPI specifications extensions to add asynchronous callbacks on communications completion, and also user code adaptations.

Among all these works, our solution on the loss of threads issue differs from $[4,11,16]$ : we aim at no user code modifications, and to progress both communications and computations by any thread opportunistically. Our 
approach is more likely a mix of $[9,16,18]$ with automation through runtime interoperations. This part of our strategy is detailed in section 3.1.

Other reasons can lead to threads idling. For instance, an unbalanced distribution of work between nodes leads to threads idling. CHAMELEON [7] is a reactive task load balancing for MPI+OpenMP tasks applications and enables OpenMP tasks migration between MPI processes. Another idling reason could be communication synchronization. A dynamic broadcast algorithm for task-based runtimes was proposed in [5] which aims at no synchronizations. Their algorithm consists of aggregating data-send operations to a single request, which holds all the recipients' information. Our work assumes that the task load is balanced across MPI processes and that applications only use asynchronous point to point communications. Thus, our scheduling strategy should be used alongside $[5,7]$ to achieve the best performances in real-life applications.

Asynchronous communications progression in hybrid MPI + OpenMP tasks programming is discussed by David Buettner et al. in [2]. They proposed an OpenMP extension to mark tasks that contain MPI communications. This allows them to asynchronously progress MPI communication on every OpenMP scheduling point. We retrieved this technique in our paper, as part of our execution model. However, we propose it without the need to mark tasks on the user side, by adding runtime interoperability.

\section{Task Scheduling Strategy}

We target applications that nest MPI point-to-point communications within OpenMP dependent tasks. Our strategy aims at scheduling first tasks that send data to reduce idle time on the receiving side. For this purpose, let us denote respectively recv-tasks and send-tasks tasks that contain MPI_Recv and MPI_Send calls, or their non-blocking version - MPI_Irecv and MPI_Isend - paired with a MPI_Wait. We will discuss in Section 3.3 how to identify them. This section starts by the presentation of the assumed interoperation between OpenMP and MPI. Then it exposes, in a progressive way, different policies to adapt task scheduling in order to send data at the earliest: through manual (using OpenMP priority clause) or automatic computation of the required tasks annotation.

\subsection{Interoperation between MPI and OpenMP runtimes}

Each MPI process has its own OpenMP scheduler, which executes tasks according to their precedence constraints (expressed through the depend clause) and their priorities. To address the loss of cores issue introduced by a blocking MPI communication calls, and to keep asynchronous communication progression, we propose a mix of User Level Threads (ULT), TAMPI, and MPI_Detach $[2,9,16,18]$. On the MPI runtime side, whenever a thread is about to block, it injects a communication progression polling function inside 
OpenMP, to be called on every scheduling point - as it was proposed by D. Buettner and al. [2]. Moreover, the MPI runtime notifies the OpenMP runtime that the current task must be suspended. The MPI communication progresses on each OpenMP scheduling point, and whenever it completes, MPI notifies OpenMP runtime that the suspended task is eligible to run again. This solution enables the progression of both communication and computation asynchronously, by any threads opportunistically.

\subsection{Manual policies}

This section presents a preliminary strategy to favor communication tasks based on source-code modification to evaluate the overall approach. To express the fact that a specific task contains an MPI communication or it is on a path to such operation, this strategy relies on the OpenMP priority clause. Thus, this section introduces 3 manual policies to annotate programs in order to fix the priorities of each task. We call this process TDG prioritization: Figure 3 resumes the priorities setting by the policies.

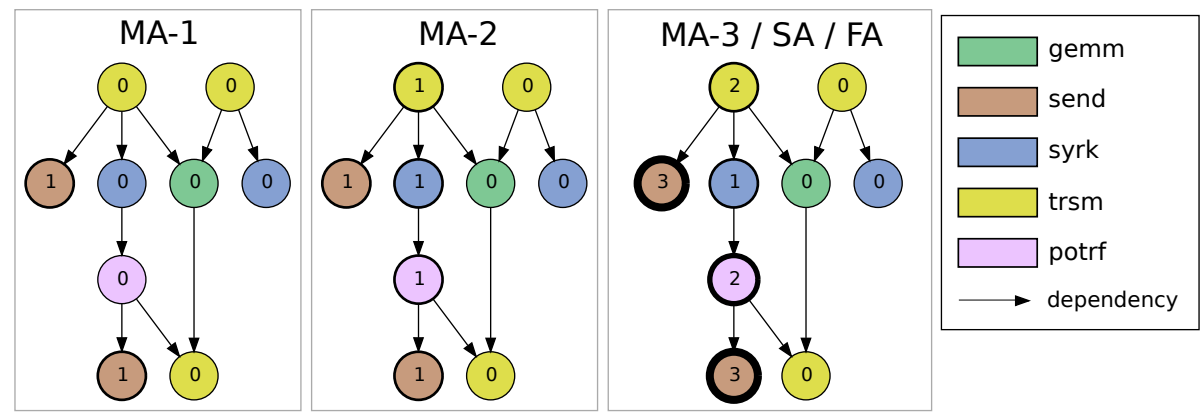

Fig. 3. Task priorities set by the various policies for the graph Figure 1 - the value on the nodes corresponds to the task priority

MA-1 relies on a binary priority 0 (the lowest) and 1 (the highest). Tasks with priority 1 are always scheduled - once dependencies are fulfilled - over the ones with priority 0 . Here, the user has to manually sets priority 1 on send-tasks, and sets no priority on other tasks resulting in a 0 low priority internally. This way, whenever the scheduler has multiple tasks marked as ready (i.e., with fulfilled dependencies), it may schedule send-tasks if there are any. This manual prioritization policy is presented in [17].

MA-2 In similar situations to the one depicted in Figure 1, we would also like to prioritize all the tasks that precede a send-task, to fulfill its dependencies to 
the earliest. In this specific case, the user should prioritize the path ( $L, M, N$ ) to resolve 0 dependency constraint. MA-2 consists in setting to 1 the priority on send-tasks and on all their predecessors recursively.

MA-3 MA-2 does not allow to finely distinguish send-tasks and their path. However, this is important since sent data may be needed by a remote node earlier or later. The earlier the data is needed in a remote rank, the earlier it should be sent. MA-3 relies on discrete priorities to prioritize various send-tasks distinctly. One way is to use their depth in the local TDG: the shorter the path to the send-task in the local TDG, the higher the priorities on its paths. This prioritization follows Algorithm 1 and is illustrated in Figure 3. Send-tasks are set with the maximum priority (line 3). If the task has no successor (lines 4-5) or if no path from it leads to a send-task (lines 8-10), then we set no priority for it. Otherwise, there is a path from the current task to a send-task and we set its priority by decrementing the value of the highest priority among its successors (line 12).

In practice, the MA-3 strategy requires the user to annotate every task on a path that contains a send-task.

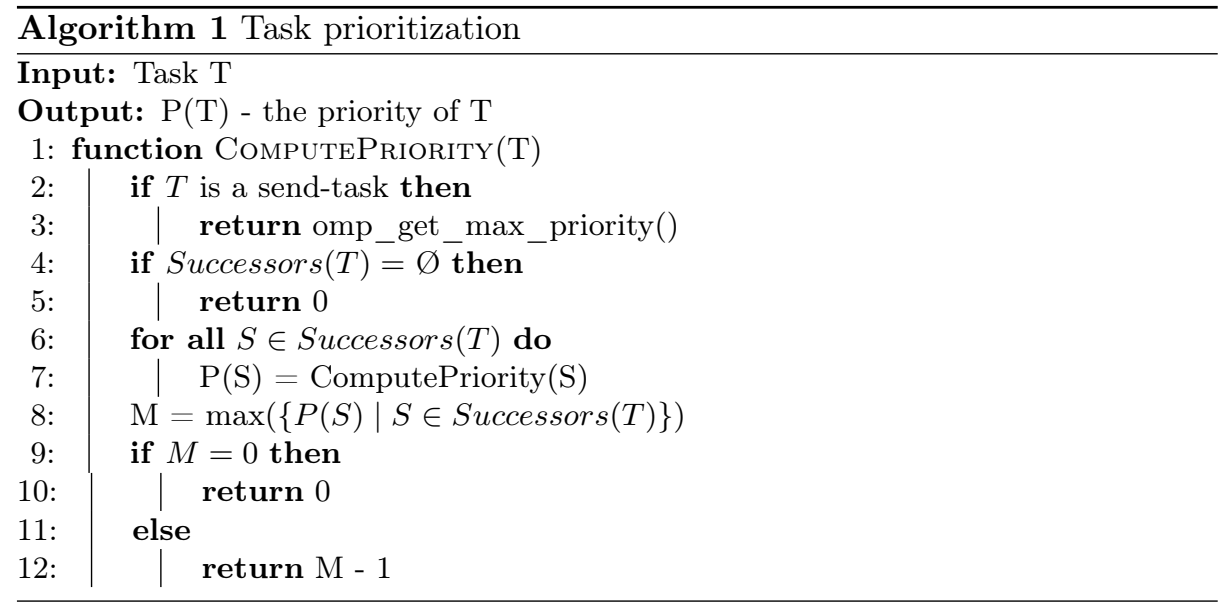

\section{3 (Semi-)Automatic Policies}

While MA-3 reduces the idling periods by sending data to the earliest, manually prioritizing the TDG is tedious to implement at user level. Users will have to manually compute the depth of each tasks in the local TDG, and setting the priority clause accordingly. From the runtime point of view, this information could be tracked. So, we propose two runtime automations on the TDG prioritization to reduce user programming efforts to identify predecessors of a task as well as to identify communication tasks. 
SA In the Semi-Automatic (SA) approach, the user simply marks send-tasks with an arbitrary priority. Once a task construct is encountered, the runtime is guaranteed that all of its predecessors were already created too. So, the runtime internally sets its priority to the highest value, and automatically propagates it through the TDG following Algorithm 1.

FA SA enables a more straightforward MA-3 implementation but it still requires the user to mark send-tasks. The Fully-Automatic (FA) approach enables a TDG prioritization similar to MA-3, but with absolutely no hints given by the users to the runtime, by adding fine collaboration between MPI and OpenMP runtimes. At execution time, whenever MPI is about to perform a send operation, it notifies the OpenMP runtime. If the current thread was executing an explicit task, it registers its profile with information such as its:

- size (shared variables)

- properties (tiedness, final-clause, undeferability, mergeability, if-clause)

- parent task identifier (the task that spawns current task)

- number of predecessors (fully-known at run-time)

- number of successors (may be incomplete)

Then, future tasks may be matched with registered profiles to detect send-tasks. This approach uses full-matching on the size, the properties, the parent task identifier, and the number of predecessors. It mainly targets iteration-based applications, where send-tasks profiles are likely to be identical between iterations.

The send-tasks cannot be detected until a task with a similar profile was scheduled, performed an MPI send operation, and registered its profiles in the OpenMP runtime. So unlike the SA policy, the prioritization cannot be done on task constructs with FA. We propose to perform it asynchronously during idle periods. This way, the runtime is more-likely to have detected send-tasks when performing the matching, and idle periods are overlapped by the prioritization without slowing down ready computations. Algorithm 2 is a single-threaded asynchronous TDG prioritization proposal. The parameter ROOTS corresponds to the task-nodes from which the prioritization should start, i.e. the blocking tasks. Line 9 to 17 consists of breadth-first-searching leaves, so we have them sorted by their depth in the TDG. Line 18 to 24 goes up from founded leaves to roots, matching tasks with registered profiles and propagating the priority to predecessors.

Table 1. Summary of Approaches

\begin{tabular}{|c|c|c|c|c|c|}
\hline Policies & MA-1 & MA-2 & MA-3 & SA & FA \\
\hline send-tasks & $\mathrm{u}$ & $\mathrm{u}$ & $\mathrm{u}$ & $\mathrm{u}$ & $\mathrm{r}$ \\
\hline send-tasks path & $\mathrm{N} / \mathrm{A}$ & $\mathrm{u}$ & $\mathrm{u}$ & $\mathrm{r}$ & $\mathrm{r}$ \\
\hline
\end{tabular}

$\mathbf{u}$ - user / manual

$\mathbf{r}$ - runtime / automatic 


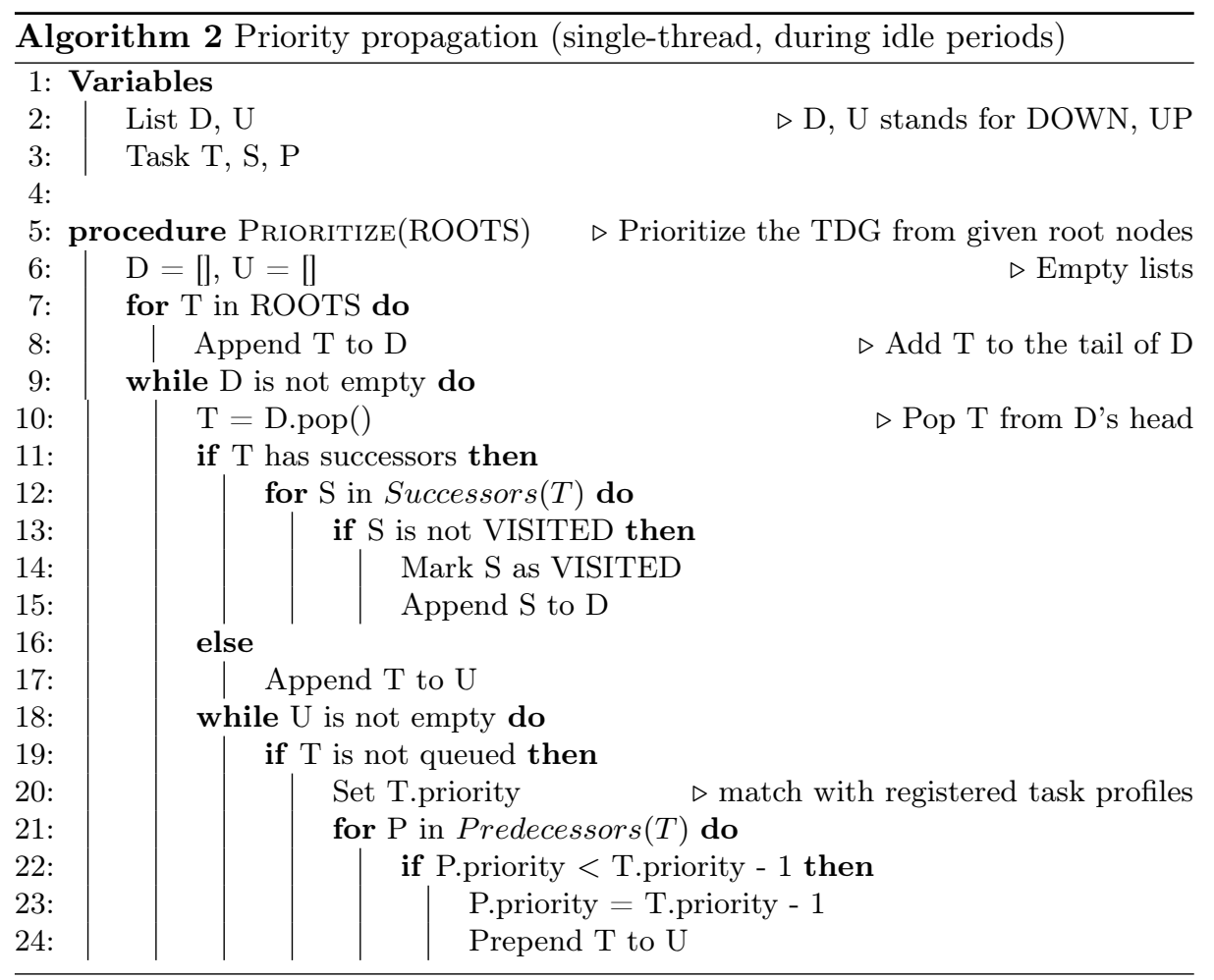

\subsection{Summary}

Table 1 summarizes the different approaches of computing priorities on tasks that perform MPI communications and tasks that contribute to execute communications (through OpenMP task dependencies). Almost all policies require user modifications of the application source code (through priority OpenMP clause) to mark tasks that perform send operations except the FA strategy that automatically detects such tasks by comparing profiles with previously-executed tasks. Furthermore, marking the whole path from the source task to the ones that perform MPI operations can be done manually (approaches MA2 or MA3) or automatically (approaches SA or FA).

\section{Implementation and Evaluation}

\subsection{Implementation}

The scheduling strategy and the different policies presented in Section 3 were implemented into MPC [15]: a unified runtime for MPI and OpenMP ${ }^{4}$. It is based on hierarchical work-stealing, similar to $[13,21]$, where red-black tree

\footnotetext{
${ }^{4}$ Available at: http://mpc.hpcframework.com/
} 
priority queues are placed at the different topological levels built from the hardware topology [10]. Each thread is assigned to multiple queues, and steal tasks from other queues when it falls idle. The task with the highest priority is popped from the selected queue.

To implement the interoperability approach presented Section 3.1, we made two modifications to MPC framework. First of all, we added an MPC-specific OpenMP entry-point to suspend the current task until an associated event is fulfilled - mpc_omp_task_block(omp_event_t event). When a thread is about to block on an MPI call, it suspends its current task through this routine. The communication progresses, and completion, omp_fulfill_event (omp_event_t event) is called so that the associated task is eligible to resume.

Furthermore, to avoid deadlocks, we added contexts to OpenMP tasks in MPC based on a modified version of the <ucontext> $\mathrm{C}$ library to handle MPC-specific TLS [3]. This add some extra instructions on tasks management measured using Callgrind. For each task, contexts added $\sim 200$ instructions on launch, $\sim 2000$ instructions on the first suspension, and $\sim 200$ instructions each time a task suspends.

Based on these modifications, we implemented the MA-1, MA-2, SA and FA approaches presented in the previous section. However, MA-3 was not implemented since it is too tedious on the user-side.

Task prioritization is done synchronously on task construct in the SA policy. In the FA policy it is done asynchronously: whenever a thread falls idle, it runs Algorithm 2 with ROOTS being the list of tasks suspended through mpc_omp_task_block.

\subsection{Evaluation Environment}

We present the result of multiple experiments on the fine-grained blocked Cholesky factorization benchmark. We denote $n$ the size of the matrix to factorize, and $b$ the size of the blocks. The time complexity of the factorization is $O\left(n^{3}\right)$, and the memory used is about $8 n^{2}$ bytes.

All experiments run onto Intel Skylake nodes (two 24-core Intel(R) Xeon(R) Platinum $8168 \mathrm{CPU} @ 2.70 \mathrm{GHz}$, with $96 \mathrm{~GB}$ of DDR). Interconnection network is a Mellanox ConnectX-4 (EDR 100Gb/s InfiniBand) system. MPC (commit 702ce5c2) was configured with optimizations enabled, and compiled with GCC 7.3.0. We forked J. Schuchart fine-grained blocked Cholesky factorization benchmark. ${ }^{5}$ The benchmark is compiled with MPC patched GCC [3], linked with Intel Math Kernel Library (MKL 17.0.6.256), MPC-MPI, and MPC-OpenMP. Each run uses SLURM exclusive parameter, which ensures that no other jobs may run concurrently on the same node. Each time corresponds to medians taken on 20 measurements.

To evaluate our strategy and different policies of Section 3 we compare measured performance against the FIFO reference policy, previously explained

\footnotetext{
${ }^{5}$ Sources are available at: https://gitlab.inria.fr/ropereir/iwomp2021
} 
in Section 1. This policy does nothing except that the MPC MPI and OpenMP runtimes interoperate to avoid deadlocks.

Fig. 4. Cholesky factorisation time based on the prioritization policy, and the MPI/OMP cores spreading on 16 Skylakes nodes (e.g., 16-48 stands for 16 MPI ranks of 48 threads each) - with a matrix of size $n=131072$, and blocks of size 512 .

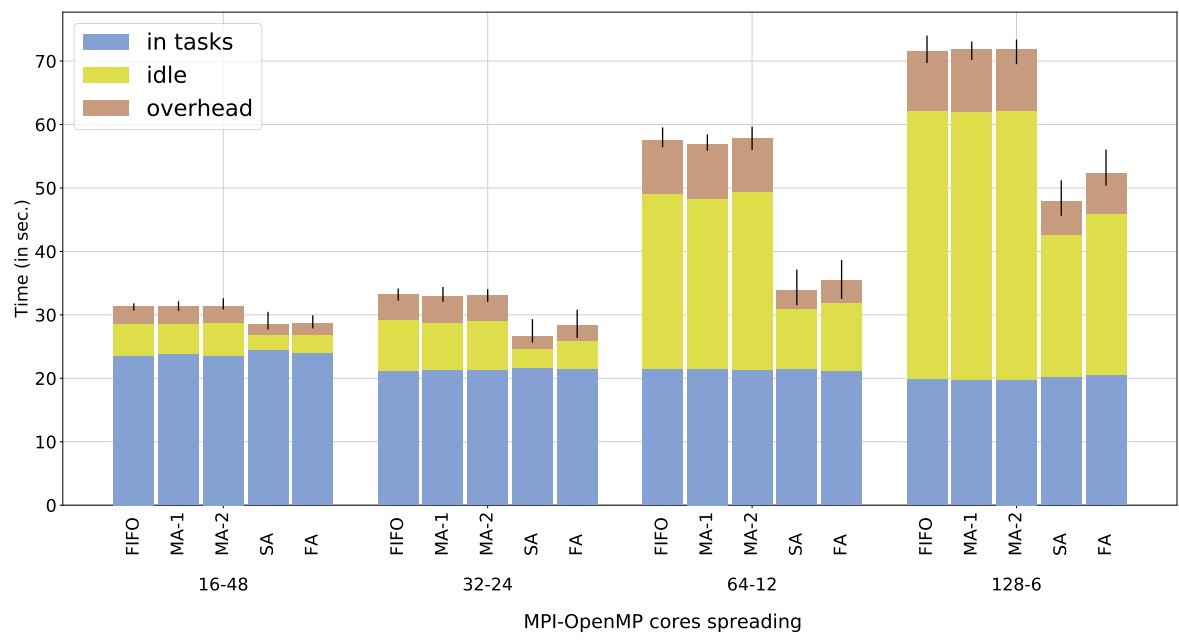

\subsection{Experimental Results}

Figure 4 shows the impact of cores spreading between MPI and OpenMP on the performance of each prioritization policy described in Section 3. The time was measured using a tool that traces every MPC-OpenMP tasks events and replay the schedule post-mortem to extract in-tasks (time spent in tasks body - MKL computation, non-blocking MPI communications initialization), idle (time spent outside tasks with no ready-tasks - idling, communications progression, prioritizations in FA), and overhead categories (time spent outside tasks with ready-tasks - tasks management, communications progression). The matrix size is $n=131072$, and 16 fully-allocated Skylake nodes. The benchmark ran with different spreading configurations. On the left-most bars, there are 16 MPI ranks of 48 OpenMP threads each (1 MPI rank per node). On the right-most bars, there are 128 MPI ranks of 6 threads each (8 MPI ranks per node). In configurations with multiple MPI ranks on the same node, OpenMP threads of the same MPI rank always are on the same NUMA node. Note that the amount of computation between each configuration remains constant, there are precisely 2.829.056 OpenMP computation tasks in each run 
distributed across MPI ranks. The number of communication tasks increases with the number of MPI ranks and is depicted in Table 2.

Table 2. Number of point-to-point communication tasks in Figure 4 runs

\begin{tabular}{|l|c|c|c|c|}
\hline Cores spreading (MPI-OpenMP) & $16-48$ & $32-24$ & $64-12$ & $128-6$ \\
\hline P2P communication tasks (overall) & 388.624 & 640.632 & 892.640 & 1.372 .880 \\
\hline
\end{tabular}

This result demonstrates that tasks prioritization in MPI+OpenMP tasks applications can have significant impact on performance (as predicted in Figures 1 and 2). First of all, MA-1 and MA-2 policies are not sufficient and they do not improve performance over the baseline FIFO policy. By prioritizing send-tasks and their path, the policies SA and FA significantly reduce idle periods. For instance in the 32-24 spreading, the total execution time and the idle time respectively are $33.2 \mathrm{~s}$ and $8.0 \mathrm{~s}$ for the FIFO policy, $28.4 \mathrm{~s}$ and $4.4 \mathrm{~s}$ for FA policy. However, being fully automatic has some costs. The FA policy is never as good as the SA one, with up to a $8 \%$ overhead in the 128-6 spreading. For FA, the prioritization only occurs once some send-tasks execution, their profile is registered and eventually a thread becomes idle to set and propagate the task priority. It means there is no prioritization for the first executed tasks. Moreover, the profile registering and matching mecanisms induce some overhead.

The 32-24 scheme reaches the best performance thanks to NUMA domain data-locality. MPC-MPI also optimizes intra-node rank exchanges, processing them in shared memory.

Table 3. Execution times of runs in Figure 5

\begin{tabular}{|l|c|c|c|c|c|c|}
\hline Number of MPI ranks & 1 & 2 & 4 & 8 & 16 & 32 \\
\hline FIFO & 22.28 s. & 24.05 s. & $25.82 \mathrm{~s}$. & 26.46 s. & 31.02 s. & 33.36 s. \\
\hline SA & 22.67 s. & 24.46 s. & 24.58 s. & 24.91 s. & 25.96 s. & 27.01 s. \\
\hline FA & 22.63 s. & 24.48 s. & 25.05 s. & 25.38 s. & 26.27 s. & 27.89 s. \\
\hline
\end{tabular}

Figure 5 is a weak-scaling on MPI ranks. Each time corresponds to the time spent by MPI processes in the factorization, given by the benchmark itself. The efficiency is relative to the mono-rank execution per prioritization policy, this is why times are also given in Table 3. Each MPI process fills a Skylake processor, with 24 OpenMP threads. The scaling starts from a single processor on 1 node, with a matrix factorization of size $n=41286$, which represents $13 \%$ of the node memory capacity. The scaling ends at 16 nodes, with 32 MPI ranks, and a matrix of size $n=131072$. The exact number of tasks is given in table 4 , where 
Fig. 5. Weak-scaling on MPI ranks, on the blocked Cholesky factorisation, with blocks of size 512, and MPI processes with 24 OMP threads per rank (Skylake socket)

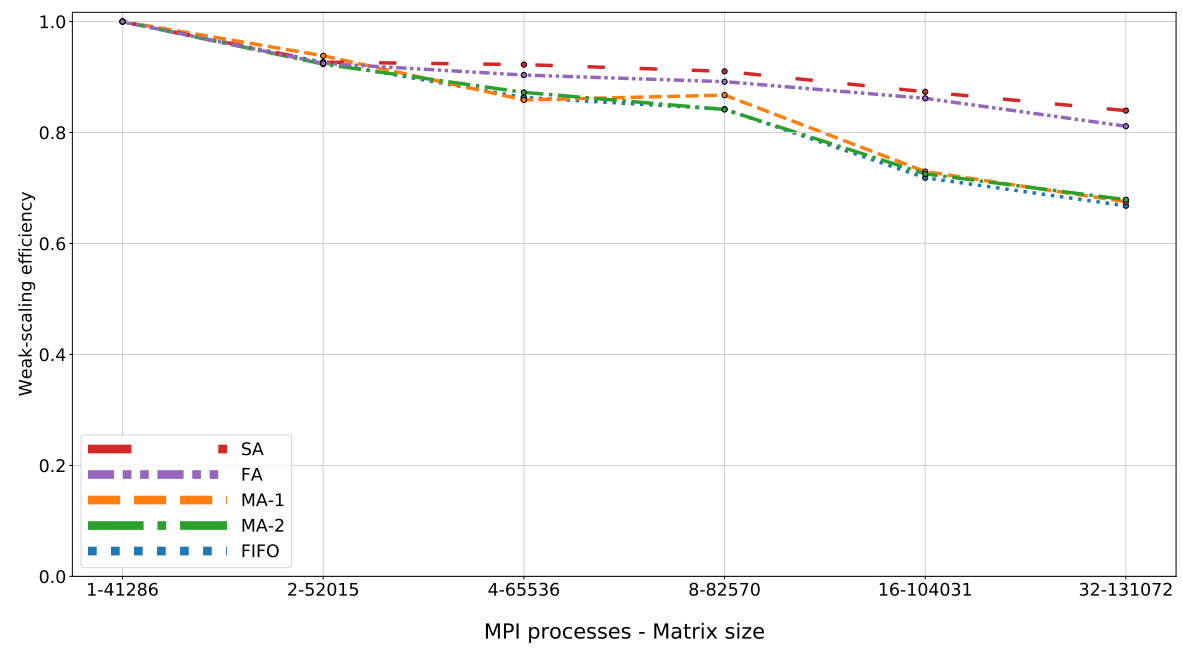

the compute category corresponds to potrf, gemm, syrk and trsm tasks, and the communication category to send-tasks and recv-tasks.

Table 4. Figure 5 tasking details

\begin{tabular}{|l|c|c|c|c|c|c|}
\hline Number of ranks & 1 & 2 & 4 & 8 & 16 & 32 \\
\hline Matrix size (n) & 41.286 & 52.015 & 65.536 & 82.570 & 104.031 & 131.072 \\
\hline Number of computation tasks & 88.560 & 176.851 & 357.760 & 708.561 & 1.414 .910 & 2.829 .056 \\
\hline Number of communication tasks & 0 & 10.100 & 32.512 & 102.082 & 243.616 & 640.632 \\
\hline
\end{tabular}

In this application, the tasks graph is evenly distributed across MPI ranks. Data-dependencies are whether retrieved from local compute tasks or through recv-tasks completion. The weak-scaling result depicted in Figure 5 scales the number of communications, while keeping constant computation work per MPI-rank. This experiment amplifies the inter-node data exchanges, and thus, the idling phenomenon we have introduced. We see that MA-1 and MA-2 prioritization does not improve the performance scaling compared to the reference FIFO prioritization. SA and FA prioritization enables better performance scaling, with up to $19 \%$ performance gain in the 32 ranks configuration. 


\section{Conclusion and Future Work}

MPI + OpenMP task programming encounters some interoperability issues that lead to thread idling. Solutions were proposed to address the loss of cores, the load balancing, or the communication collectives, but scheduling issues remain. This paper proposes a task scheduling strategy for OpenMP schedulers to reduce idle periods induced by MPI communications, by favoring send-tasks. We propose and evaluate several policies from purely manual approaches which require user cooperation to fully automatic policy.

The best method significantly improves performance and scaling of the Cholesky factorization [19], with up to $19 \%$ performance gain in our largest run. Some overhead in the fully automatic strategy has been identified and we are planning to improve graph traversal to reduce the runtime cost.

For future work, we plan to validate our approach on a wider set of applications. Furthermore, in this paper, we only considered explicit data dependencies expressed through the depend clause: we consider adding support for control dependencies. Also, prioritization of send-tasks is purely based on local information (the dependency graph between OpenMP tasks) without taking into account task dependencies in other MPI ranks. We are thinking to improve our strategy by taking into account global information.

\section{References}

1. Ayguadé, E., Copty, N., Duran, A., Hoeflinger, J., Lin, Y., Massaioli, F., Su, E., Unnikrishnan, P., Zhang, G.: A proposal for task parallelism in OpenMP. In: Chapman, B., Zheng, W., Gao, G.R., Sato, M., Ayguadé, E., Wang, D. (eds.) A Practical Programming Model for the Multi-Core Era. pp. 1-12. Springer Berlin Heidelberg, Berlin, Heidelberg (2008)

2. Buettner, D., Acquaviva, J.T., Weidendorfer, J.: Real asynchronous MPI communication in hybrid codes through OpenMP communication tasks. pp. 208215 (12 2013). https://doi.org/10.1109/ICPADS.2013.39

3. Carribault, P., Pérache, M., Jourdren, H.: Thread-local storage extension to support thread-based MPI/OpenMP applications. In: Chapman, B., Gropp, W., Kumaran, K., Müller, M. (eds.) OpenMP in the Petascale Era, Proceedings of the 7th International Workshop on OpenMP (IWOMP 2011), Lecture Notes in Computer Science, vol. 6665, pp. 80-93. Springer Berlin Heidelberg (2011). https://doi.org/10.1007/978-3-642-21487-5_7

4. Chatterjee, S., Tasırlar, S., Budimlic, Z., Cavé, V., Chabbi, M., Grossman, M., Sarkar, V., Yan, Y.: Integrating asynchronous task parallelism with MPI. In: 2013 IEEE 27th International Symposium on Parallel and Distributed Processing. pp. 712-725 (2013). https://doi.org/10.1109/IPDPS.2013.78

5. Denis, A., Jeannot, E., Swartvagher, P., Thibault, S.: Using Dynamic Broadcasts to improve Task-Based Runtime Performances. In: Euro-Par - 26th International European Conference on Parallel and Distributed Computing. Euro-Par 2020, Rzadca and Malawski, Springer, Warsaw, Poland (Aug 2020). https://doi.org/10.1007/978-3-030-57675-2_28, https://hal.inria.fr/hal-02872765

6. GNU Project: GOMP - An OpenMP implementation for GCC, https://gcc.gnu.org/projects/gomp/ 
7. Klinkenberg, J., Samfass, P., Bader, M., Terboven, C., Müller, M.: CHAMELEON: Reactive load balancing for hybrid MPI + OpenMP taskparallel applications. Journal of Parallel and Distributed Computing 138 (12 2019). https://doi.org/10.1016/j.jpdc.2019.12.005

8. LLVM Project: OpenMP®: Support for the OpenMP language, https://openmp.llvm.org/

9. Lu, H., Seo, S., Balaji, P.: MPI+ULT: Overlapping communication and computation with user-level threads. pp. 444-454 (08 2015). https://doi.org/10.1109/HPCC-CSS-ICESS.2015.82

10. Maheo, A., Koliaï, S., Carribault, P., Pérache, M., Jalby, W.: Adaptive OpenMP for large NUMA nodes. pp. 254-257 (06 2012). https://doi.org/10.1007/978-3-64230961-8 720

11. Marjanovic, V., Labarta, J., Ayguadé, E., Valero, M.: Effective communication and computation overlap with hybrid MPI/smpss. vol. 45, pp. 337-338 (05 2010). https://doi.org/10.1145/1693453.1693502

12. Meadows, L., Ishikawa, K.i.: OpenMP tasking and MPI in a lattice qcd benchmark. pp. 77-91 (08 2017). https://doi.org/10.1007/978-3-319-65578-9_6

13. Olivier, S.L., Porterfield, A.K., Wheeler, K.B., Spiegel, M., Prins, J.F.: OpenMP task scheduling strategies for multicore NUMA systems. The International Journal of High Performance Computing Applications 26(2), 110-124 (2012). https://doi.org/10.1177/1094342011434065

14. OpenMP Architecture Review Board: OpenMP application program interface version 3.0 (May 2008), http://www.openmp.org/mp-documents/spec30.pdf

15. Pérache, M., Jourdren, H., Namyst, R.: Mpc: A unified parallel runtime for clusters of NUMA machines. In: Proceedings of the 14th International Euro-Par Conference on Parallel Processing. p. 78-88. Euro-Par '08, Springer-Verlag, Berlin, Heidelberg (2008). https://doi.org/10.1007/978-3-540-85451-7_9

16. Protze, J., Hermanns, M.A., Demiralp, A., Müller, M.S., Kuhlen, T.: MPI detach asynchronous local completion. In: 27th European MPI Users' Group Meeting. p. 71-80. EuroMPI/USA '20, Association for Computing Machinery, New York, NY, USA (2020). https://doi.org/10.1145/3416315.3416323

17. Richard, J., Latu, G., Bigot, J., Gautier, T.: Fine-Grained MPI+OpenMP Plasma Simulations: Communication Overlap with Dependent Tasks. In: EuroPar 2019: Parallel Processing - 25th International Conference on Parallel and Distributed Computing. pp. 419-433. Springer, Göttingen, Germany (Aug 2019). https://doi.org/10.1007/978-3-030-29400-7_30, https://hal-cea.archivesouvertes.fr/cea-02404825

18. Sala, K., Teruel, X., Perez, J.M., Peña, A.J., Beltran, V., Labarta, J.: Integrating blocking and non-blocking MPI primitives with taskbased programming models. Parallel Computing 85, 153-166 (2019). https://doi.org/10.1016/j.parco.2018.12.008

19. Schuchart, J., Tsugane, K., Gracia, J., Sato, M.: The impact of taskyield on the design of tasks communicating through MPI. In: de Supinski, B.R., Valero-Lara, P., Martorell, X., Mateo Bellido, S., Labarta, J. (eds.) Evolving OpenMP for Evolving Architectures. pp. 3-17. Springer International Publishing, Cham (2018)

20. Seo, S., Amer, A., Balaji, P., Bordage, C., Bosilca, G., Brooks, A., Carns, P., Castelló, A., Genet, D., Herault, T., Iwasaki, S., Jindal, P., Kalé, L.V., Krishnamoorthy, S., Lifflander, J., Lu, H., Meneses, E., Snir, M., Sun, Y., Taura, K., Beckman, P.: Argobots: A lightweight low-level threading and tasking framework. IEEE Transactions on Parallel and Distributed Systems 29(3), 512-526 (2018). https://doi.org/10.1109/TPDS.2017.2766062 
21. Virouleau, P., Broquedis, F., Gautier, T., Rastello, F.: Using data dependencies to improve task-based scheduling strategies on NUMA architectures. In: EuroPar 2016. Euro-Par 2016, Grenoble, France (Aug 2016), https://hal.inria.fr/hal01338761

22. Yang, T., Gerasoulis, A.: Dsc: Scheduling parallel tasks on an unbounded number of processors. Parallel and Distributed Systems, IEEE Transactions on 5, 951 967 (10 1994). https://doi.org/10.1109/71.308533 